\title{
La politique européenne de voisinage, un complexe de sécurité à géométrie variable
}

The European Neighbourhood policy, a security complex of variable geometry

\section{Thierry Balzacq}

\section{(2) OpenEdition}

12 Journals

\section{Édition électronique}

URL : http://journals.openedition.org/conflits/2481

DOI : 10.4000/conflits.2481

ISSN : $1777-5345$

Éditeur :

CCLS - Centre d'études sur les conflits lilberté et sécurité, L'Harmattan

\section{Édition imprimée}

Date de publication : 17 septembre 2007

Pagination : 31-59

ISBN : 978-2-296-03458-7

ISSN : 1157-996X

\section{Référence électronique}

Thierry Balzacq, «La politique européenne de voisinage, un complexe de sécurité à géométrie variable », Cultures \& Conflits [En ligne], 66 | été 2007, mis en ligne le 13 mars 2008, consulté le 30 mars 2021. URL : http://journals.openedition.org/conflits/2481; DOI : https://doi.org/10.4000/conflits.2481 


\section{La politique européenne de voisinage, un complexe de sécurité à géométrie variable 1}

\section{Thierry BALZACQ}

Thierry Balzacq est professeur de science politique à l'université de Namur en Belgique et il enseigne la théorie des relations internationales à Sciences Po Paris. Il est chercheur associé an Centre for European Policy Studies (CEPS) de Bruxelles et au Centre d'études des crises et conflits internationaux (CECRI) de l'université catholique de Louvain. Ses travaux portent sur les approches critiques de la sécurité, la théorie des relations internationales et les effets de la démarcation géographique sur la politique extérieure de l'UE.

T 'élargissement de décembre 2004 a soulevé, pour l'UE, des questions stratégiques redoutables. Comment canaliser - et endiguer - les aspirations des uns et des autres à l'adhésion, sans compromettre la qualité des relations qu'elle souhaite entretenir avec les nouveaux Etats voisins ? Comment, dans ces conditions, construire une communauté de sécurité pluraliste à l'intérieur de l'espace de l'Union, sans que les voisins ne se sentent exclus ou - ce qui charrie des conséquences similaires - menacés ? Enfin, comment impliquer ces derniers dans la gestion des questions cruciales pour l'UE (immigration illégale, crime organisé, terrorisme, énergie) sans que cela ne soit vécu comme de l'ingérence ? De telles questions ne sont évidemment pas neutres par rapport à l'idée plus ou moins précise de ce que l'UE voudrait être, bien qu'elles ne se confondent pas avec elle. En effet, pour le dire autrement, l'élargissement n'a pas fait qu'intégrer de nouveaux Etats-membres, elle a aussi eu pour résultat immédiat d'instituer des lignes de séparation inédites et, par la suite, de porter les frontières externes de l'Union, à l'Est essentiellement, au contact d'une constellation de voisins jugés différents du point de vue économique, social et politique.

1. Ce texte a bénéficié du formidable instrument d'apprentissage qu'est l'évaluation anonyme par des pairs. Il est également, à certains égards, le fruit des discussions eues avec les participants à la première école doctorale CHALLENGE - The Changing Landscape of European Liberty and Security - (6 $6^{\mathrm{e}}$ PCRD) qui s'est tenue au Centre for European Policy Studies (CEPS) à Bruxelles les 21 et 22 avril 2006. 
L'ensemble de ces constats montre combien la PEV doit nombre de ses caractéristiques centrales aux différences qui affectent, dans ses structures même, la politique extérieure de l'UE. Cerner ces différences suppose que l'on se donne pour projet d'examiner les effets du clivage géographique sur les rapports de l'Union à ses voisins. Or, le but initial de la Politique européenne de voisinage (PEV) est de réguler les externalités négatives de sécurité nées de l'élargissement ${ }^{2}$. L'ex-commissaire aux Relations extérieures, Christopher Patten, et Javier Solana, le Haut Représentant pour la PESC soulignent dans une lettre conjointe que "l'élargissement pose un double défi, celui d'obvier à la formation de nouvelles lignes de démarcation en Europe tout en apportant une réponse aux attentes suscitées par les frontières nouvellement créees de l'Union ${ }^{3}{ }^{»}$.

Cet article a pour objet l'examen des processus et effets géographiques de la PEV. L'analyse qui suit tient à des choix accomplis a priori, dont il faudrait d'emblée établir la logique pour éviter que l'on recherche dans ce texte ce qui ne s'y trouve point. Il faut souligner tout d'abord qu'on ne s'intéressera pas à la production du contenu de la PEV - c'est un des aspects qu'aborde Julien Jeandesboz dans ce numéro - qui, en dépit de sa fécondité évidente, nous conduirait à dévier considérablement cette étude de son axe d'orientation ${ }^{4}$. On s'attachera en revanche aux principes - conditionnalité et socialisation - qui structurent le contenu de la PEV et organisent le type de relations que l'UE entend entretenir avec ses voisins. On ne cherchera pas non plus à dégager, comme le suggèrent certains travaux, les implications globales de la PEV. Une telle entreprise nous semble en effet soit trop optimiste quant à la portée réelle de la $\mathrm{PEV}$, soit démesurée ${ }^{5}$. Notre ambition est résolument plus modérée. On tentera de saisir les effets régionaux des principes auxquels a recours la PEV pour inscrire son projet dans la pratique. Enfin, sur le plan théorique, on se limitera, pour éviter un investissement conceptuel superflu, à l'essentiel des idées du constructivisme qui nous permet de mener à son terme la tâche qui nous incombe ${ }^{6}$. En un mot, on fera nôtre cet aphorisme de

2. Les documents officiels et scientifiques sur la PEV se trouvent sur la page suivante : http :// ec.europa/world/enp/documents_fr.htm. L'auteur a consulté les documents, soit en anglais, soit en français, cela notamment à cause des différents environnements de travail au sein desquels il a mené ses recherches sur divers aspects de la PEV. Les traductions de l'anglais vers le français ont été assurées par l'auteur.

3. Patten C., Solana J., Wider Europe, Bruxelles, 7 août 2002. Texte disponible sur : http//:europa.eu.int/comm/world/enp/pdf/_0130163334_001en.pdf

4. Ce contenu touche des domaines aussi variés que justice et affaires intérieures, économie, sécurité et régulation du marché de l'énergie, environnement, liberté de la presse, démocratisation, égalité des genres, droits de l'Homme. Tous les plans d'actions couvrent la plupart de ces secteurs. Voir par exemple le Plan d'action UE-Maroc (2004) et le Plan d'action UE-Ukraine (2004).

5. Voir par exemple Aliboni R., "The geopolitical implications of the European Neighbourhood Policy ", European Foreign Affairs Review, vol.10, 2005, pp. 1-16.

6. Ici, comme dans ce qui suit, nous nous appuyons sur un constructivisme de type rationaliste. Sur cette approche voir Wendt A., Social theory of international politics, Cambridge, Cambridge University Press, 1999. Ce choix se justifie d'autant plus que Buzan et l'école de Copenhague s'y réfèrent pour construire leur modèle. Voir Buzan B., Wrever O., de Wilde J., Security: A New 
Raymond Aron : «les modèles théoriques des relations internationales ne servent et ne doivent servir qu'à préparer l'étude concrète ${ }^{7}$ ».

En tant qu'un des volets les plus originaux de la politique extérieure de l'Union, les buts de la PEV ont évolué substantiellement depuis la lettre de Patten et Solana. Il s'agit en effet de promouvoir et de soutenir une série de réformes politiques, économiques et sociales, essentiellement à travers l'insertion des voisins au sein du cadre normatif communautaire. En cela, la perspective esquissée par la PEV peut être rapprochée des pratiques se rattachant aux Etats de l'espace économique européen. Toutefois, contrairement à ces derniers qui pourraient, s'ils le souhaitaient, devenir membres de l'UE, les partenaires de la PEV constituent un groupe assez hétérogène qui, au regard des perspectives d'adhésion, n'ont ni les mêmes attentes ni les mêmes possibilités. Reconnaissant que «l'Union ne peut s'élargir indéfiniment 8 ", la PEV est l'espace privilégié où se trame une forme plus subtile, plus flexible, plus difficile à coordonner, de gestion des frontières externes de l'UE.

L'objectif de cet article n'est donc pas, on l'aura compris, de faire état de «manière totalisante 9 » des dimensions géographiques de la PEV. En revanche, l'étude se situera à ce point névralgique de la PEV où l'exigence de sécurité interne rejoint la nécessaire institutionnalisation des frontières et - par accident ou par projet - débouche sur un complexe de sécurité à géométrie variable. Après avoir défini le socle théorique, nous examinerons le rapport des complexes de sécurité avec la fabrication de la frontière - la frontiérisation. Le chemin sera alors libre pour montrer comment la gestion des frontières notamment à travers la gouvernance externe - conduit à une situation paradoxale : l'inclusion par la différenciation. Nous pourrons, enfin, délimiter les propriétés élémentaires qui caractérisent chacune des régions constitutives de la PEV. Au regard de ces éléments, l'ordre d'exposition adopté a pour effet de montrer en quelque sorte l'extraordinaire imbrication de processus et facteurs qu'il n'est pas rare que la littérature sur la PEV tienne pour séparés.

\section{De la sécurité régionale au complexe de sécurité}

La question de savoir quelles sont les spécificités du niveau régional de la sécurité revient à sonder, au moins brièvement, l'idée de « région » en Relations internationales ${ }^{10}$. Pour cette discipline, en effet, la région se décline de manière

Framework for Analysis, Boulder, Lynne Rienner, 1998 ; Buzan B., Wxver B., Regions and Power: The Structure of International Security, Cambridge, Cambridge University Press, 2003.

7. Aron R., Paix et guerre entre les nations, Paris, Calmann-Lévy, 1984, p. 156.

8. Prodi R., $A$ Wider Europe - A Proximity Policy as the Key to Stability. Discours tenu lors du $6^{\mathrm{e}}$ ECSA World Peace Conference, "Security and Stability: International Dialogue and the Role of the EU », Bruxelles, 5-6 décembre 2002. Disponible sur :

$\mathrm{http} / / /$ europa.eu.int/rapid/start/cgi/guesten.ksh?p_action.gettxt=gt\&doc=SPEECH/02/619| O|RAPID\&lg=EN\&display=

9. Dobry M., Sociologie des crises politiques, Paris, Presses de la FNSP, 1992, p. 46.

10. D’une façon générale, mais aussi du point de vue théorique, les enjeux du régionalisme sont 
duale : d'une part le volet «sub-étatique », et d'autre part le versant «supraétatique ». Le premier désigne les régions internes à l'unité de base des relations internationales : l'Etat. Ici, par essence, il n'y a donc région que parce que l'Etat, généralement suite à une révision constitutionnelle, en a décidé ainsi. Le préfixe «supra » indique, quant à lui - encore qu'il ne soit réellement approprié - précisément ceci : l'agrégation, formelle ou non, d'entités étatiques autour d'intérêts et d'identités communs. Le niveau «sub » ne s'internationalise, par ailleurs, que lorsqu'il prend corps au sein d'une institution supranationale telle que le Comité des régions au sein de l'UE. C'est pourquoi ce qui s'impose à notre examen se limite d'emblée au niveau «supra ».

Il convient d'ajouter que la définition de la région est cruciale, notamment parce qu'elle permet d'aller au-delà d'une simple description, pour saisir les facteurs essentiels qui rendent possible la manifestation d'une région. Ceci ne consiste qu'en un énoncé définissant les contours de la sécurité régionale qui procède de ce qui lui est propre : l'interaction des acteurs, la géographie et l'ontologie relationnelle, c'est-à-dire la nature sédimentée des rapports - ami, ennemi ou rival. Ce qui veut dire la chose suivante : la logique de la sécurité régionale est située au cœur d'un truisme décisif, le caractère essentiellement relationnel de la sécurité 11. De là vient le fait que seuls les rapports entre acteurs étatiques que nous n'abordons, pour des raisons d'économie analytique, qu'en termes de menaces et de vulnérabilités intersubjectives, nécessitent une imposition de formes régulatrices: une schématisation en vue de leur plus grande intelligibilité. Ceci peut ne sembler qu'une répétition de l'idée précédente. Mais nous voulons signifier davantage : les définitions de la région varient, mais les déterminants - interaction, proximité géographique et ontologie relationnelle - subsistent, en filigrane.

Nous pouvons à présent circonscrire l'enchaînement des définitions concurrentes mais complémentaires quant au contenu, et nous n'en sommes relativement capables qu'en vertu des prémisses susmentionnées, qui orientent notre démarche. Tout d'abord, un sous-système régional, au sens où l'en-

assez bien résumés par Boyd G. (ed.), Regionalism and Global Security, Lexington, Lexington Books, 1984 ; Rittenhouse J., Courtice R.G. (eds.), Regionalism and Theory, Lewigton, Edwin Mellon Press, 1992 ; Fawcett L., Hurrell A. (eds.), Regionalism in World Politics: Regional Organization and International Order, Oxford, Oxford University Press, 1995 ; Winters A.L., Regionalism versus Multilateralism, Londres, Centre for Economic Policy Research, 1996; Gamble A., Payne A. (eds.), Regionalism and World Order, Basingstoke, Macmillan 1996. Pour un état des lieux de la recherche dans le domaine du régionalisme, voir Väyrynen R., "Regionalism: old and new ", International Studies Review, vol.5, n 1,2003 , pp. 25-52.

11. Voir par exemple Hertz J., "Idealist international relations and security dilemma ", World Politics, vol.2, n², 1950, pp. 157-180; Wolfers A., Discord and Collaboration: Essays on International Politics, Baltimore, Johns Hopkins University Press, 1962 ; Jervis R., Perception and Misperception in International Relations, Princeton, Princeton University Press, 1976 ; Jervis R., "Cooperation under the security dilemma ", World Politics, vol.30, n², 1978, pp. 167-214. Sur les apports du relationnisme en théories des Relations internationales voir Jackson P.T., Nexon D., « Relations before states: substance, process and the study of world politics », European Journal of International Relations, vol.5, n³, 1999, pp. 291-332. 
tend Williams Thompson, se définit par trois éléments : (i) les attributs - la proximité géographique, la régularité et le volume des interactions, lesquelles expliquent que les variations à l'intérieur d'une unité entrainent des altérations en d'autres points du sous-système; (ii) la reconnaissance interne et externe d'un groupe d'Etats comme membres d'un espace délimité ; et (iii) la taille de cet ensemble, fonction du nombre et de la puissance cumulée des unités impliquées ${ }^{12}$. Quel que soit ici l'état des choses, il reste que Louis Cantori et Steven Spiegel proposent de définir la région en des termes quasi similaires. La région, affirment-ils, est délimitée

«-du moins en partie - par rapport aux éléments géographiques, même si les facteurs sociaux, économiques, politiques et organisationnels sont tout autant pertinents. En conséquence, les membres [d'une entité régionale] sont proches, sans être nécessairement contigus ${ }^{13} »$.

Ces variables sont réarticulées, avec plus d'ordre, par Raimo Väyrynen :

«Un sous-système régional est caractérisé par une certaine singularité et une (réelle) proximité, non seulement au sens géographique, mais aussi en termes économiques et politiques. La proximité est institutionnalisée à travers les interactions mutuelles et les organisations communes ${ }^{14}$ ».

Ainsi se trouve posé le socle propre à déterminer les variables cruciales d'une région en Relations internationales : la proximité géographique et la densité interactionnelle dont les forces conduisent progressivement à la production manifeste d'une ontologique sociale spécifique, véhicule de puissances intégratives ou dissociantes. Selon Durkheim, à qui cette idée de «densité dynamique ou interactionnelle " est empruntée, "l'accroissement $d u$ volume et de la densité dynamique des sociétés modifie fondamentalement leur existence collective ». En revanche, dès que l'on a posé cette définition, on s'aperçoit que celle-ci soulève deux questions adjacentes: (i) qu'est-ce que le

12. Thompson W.T., «The regional subsystem: a conceptual explication and a propositional inventory ", International Studies Quarterly, vol.17, n`1, 1973, p. 101. Notre vision du système s'inspire de celle de Michael Brecher qui le conçoit comme étant un «ensemble d'acteurs soumis à des contraintes intérieures (contexte) et extérieures (environnement), placés dans une configuration de pouvoir (structure) et impliqués dans des réseaux réguliers d'interactions (processus)». Voir Brecher M., «Système et crise en politique internationale », in Korany B., Analyse des relations internationales. Approches, concepts, donnés, Montréal, Gaétan Morin, $2^{\mathrm{e}}$ éd., 1987, p. 75.

13. Cantori L.J., Spiegel S. L., "The international relations of regions », in Falk R. A., Russet B. M. (éds.), International Regions and the International System, Chicago, University of Chicago Press, 1967 ; Cantori L.J., Spiegel S.L., The International Politics of Regions: A Comparative Approach, Englewood Cliffs, Prentice-Hall, 1970.

14 . Väyrynen R., "Regional conflict formation: an intractable problem of international relations ", Journal of Peace Research, vol. 21, n4, 1984, p. 340. 
volume ? Et (ii) qu'est-ce que la densité dynamique ? Par volume, Durkheim désigne le nombre d'unités sociales pertinentes. La densité dynamique, quant à elle, correspond à la quantité, la vitesse, et la diversité agrégée des transactions actives (économiques, politiques...) au sein d'une architecture sociale ${ }^{15}$. Il s'ensuit qu'au sein de la sécurité régionale, les notions de «volume » et de «densité dynamique » sont fonction l'une de l'autre, les processus qu'ils génèrent étant déterminants pour l'état du sous-système.

En délimitant ainsi la problématique, la question de la sécurité régionale apparaît métamorphosée. Elle se trame, dès lors, à l'intersection d'au moins deux phénomènes qu'il convient de distinguer ${ }^{16}$ : (i) la jonction progressive de divers champs politiques, économiques et/ou sociaux ; (ii) l'intensité des interactions externes. Cela signifie dans le même temps que la ligne principale de la sécurité régionale, c'est l'interdépendance. Toutefois, la nature de celle-ci varie en fonction de l'ontologie relationnelle qui prévaut et de la distribution des capacités (militaires et/ou économiques surtout). Cette postulation théorique, assurément fragile, conduit pourtant au district fondamental de la sécurité régionale qui inclut tant l'interdépendance positive que négative. Les deux pôles du continuum de la sécurité régionale, qui recouvrent par ailleurs la conflictualité et la coopération pures, sont indissociablement réintégrés dans le concept structurant de «complexe de sécurité ». En d'autres termes : «un groupe d'Etats dont les inquiétudes et les perceptions majeures de sécurité sont liées à un point tel que leurs problèmes de sécurité nationale ne peuvent raisonnablement être analysés ou résolus séparément 17 ". Cette définition conductrice, c'est-à-dire cette précision analytique, qui maîtrise à la fois les aspects formels et non formels de la sécurité régionale, dévoile dès le départ ce qui distingue le complexe de sécurité des autres formulations. La foncière reconnaissance des unités ${ }^{18}$, le statut subsidiaire des facteurs socio-économiques ${ }^{19}$ et l'institutionnalisation de la proximité géographique ${ }^{20}$ sont respectivement soumis à caution, amendés, ou tout simplement abandonnés. Ce qui occupe désormais le centre de l'analyse, c'est la proximité géographique couplée à l'objectivation d'une ontologie relationnelle au sein d'une institution formelle (OTAN, SADC - Southern African Development Community) ou non (par exemple, le nœud conflictuel de la région des Grands Lacs en Afrique centrale).

15. Durkheim E., Les Règles de la méthode sociologique, Paris, Alcan, 1895.

16. Ce qui est une autre façon de dire que ces conditions sont nécessaires et non suffisantes. Car dans la formation de la sécurité ou de l'insécurité régionale, il est inapproprié d'omettre la variable de rôle des élites politiques ou encore leur identité personnelle et sociale. En réalité, le développement d'une région repose (beaucoup) sur de telles variables et mériterait, à cet égard, plus d'attention.

17. Buzan B., People, States and Fear: An Agenda for International Security Studies in the Postcold War Era, Londres, Longman, 1991, p. 190 ; l'édition de 1983 contenait déjà cette définition. Voir Buzan B., People, States and Fear, 1983, p. 106. Voir aussi Buzan B., Wrever O., de Wilde J., op. cit., p. 12.

18. Thompson W.T., op. cit.

19. Cantori L.J., Spiegel S.L., op. cit.

20. Väyrynen R., op. cit. 
Le dispositif théorique mis en place par Buzan conduit à aborder le complexe de sécurité comme un entrelacement de relations géographiquement circonscrites. Le complexe de sécurité reprend ainsi le contenu saillant de la région, indiqué par Thompson, Väyrynen, Cantori et Spiegel, pour l'enchâsser dans un énoncé principiel qui, du même coup, replace originalement l'ensemble de cette spécification de la sécurité régionale à l'interface des sécurités nationale et internationale ${ }^{21}$. Cela permet de rendre compte simultanément des dynamiques endogènes du complexe entre acteurs constitutifs d'une part, et de l'impact des interventions externes, qui altèrent la configuration et la nature du complexe, d'autre part ${ }^{22}$.

\section{L'essence «structurante » du complexe régional de sécurité}

Cela dit, revenons, pour en tracer les contours et en définir les types idéaux, sur la formulation théorique des complexes de sécurité que propose Buzan. Tout d'abord, la caractérisation des complexes de sécurité n'est pas monolithique. Il est courant de distinguer une formulation réaliste et une autre, que l'on nommera « constructiviste ». La conception réaliste a pour référent de la sécurité l'Etat, et comme secteur décisif le politico-militaire. Il s'agit de déceler les rapports militaires les plus denses permettant de montrer qu'il existe une structure de sécurité régionale « visible ». Cependant, nous référant à la théorie réaliste (classique) du complexe de sécurité, ce sont d'autres éléments que nous cherchons à comprendre. Nous voulons donner un exposé concis de ses principales caractéristiques : d'abord, les complexes classiques sont composés de deux Etats au moins, lesquels constituent un conglomérat géographiquement cohérent ; ensuite, les relations entre les Etats membres du complexe sont marquées par l'interdépendance sécuritaire, laquelle peut être positive ou négative, mais significativement forte, au regard de la faiblesse des liens avec l'environnement externe au complexe; enfin, la configuration de l'interdépendance sécuritaire doit être profonde et durable, pas nécessairement permanente ${ }^{23}$.

Or, parce que ces complexes de sécurité sont ainsi établis, une chose émerge clairement : ce sont des ensembles autonomes, "micro versions $d u$ système politique international au sein duquel ils sont inscrits $24 »$. De la sorte, la plupart des complexes réalistes de sécurité, fondés sur l'étato-centrisme, sont continentaux ou subcontinentaux : Amérique du Sud, Moyen-Orient, Asie du Sud, etc. La théorie réaliste du complexe de sécurité est par conséquent extrêmement limitée. C'est en ce sens qu'il s'agit de lui substituer un cadre plus flexible et, qui plus est, d'une opérationnalité corrigée. Dans cette optique, Buzan, Wrver et de Wilde proposent deux voies, la première consistant à adopter une méthode constructi-

21. Ce rôle de médiation ou de substitution est reconnu aux organisations régionales dans le cadre de la Charte des Nations unies, chap. VIII.

22. Voir Ayoob M., The Third World Security Predicament: State Making, Regional Conflict, and the International System, Boulder, Lynne Rienner, 1995.

23. Buzan B., Wrever O., de Wilde J., Security, op. cit., p. 15.

24. Ibid. 
viste dans l'étude des questions de sécurité. Plus spécifiquement, les tenants de l'école de Copenhague plaident en faveur d'une analyse de la construction discursive des questions de sécurité : la «sécuritisation 25 ». Dans ce contexte, l'énonciation de la sécurité ne se réfère pas à une configuration externe donnée $a$ priori, mais elle réalise par elle-même une situation de sécurité. On peut dire que ce sont les caractéristiques de l'acte de parole qui modifient la nature d'un problème et l'élève au rang de priorité sécuritaire. Cela signifie que le niveau d'analyse dépend du site d'énonciation. Il reste à nous demander quelle est la deuxième stratégie permettant de se dégager d'une vision classique des complexes de sécurité. Eu égard au réalisme, l'Etat et le secteur politico-militaire constituent les pièces centrales du complexe de sécurité. En revanche, dans une vision constructiviste, en vertu de la possible construction rhétorique de l'insécurité, les secteurs de la sécurité et la nature des objets menacés peuvent être considérablement variés. Cela veut dire aussi que les complexes de sécurité sont soit homogènes, soit, en sens contraire, hétérogènes ${ }^{26}$.

Le complexe homogène se focalise sur l'interdépendance fonctionnelle des acteurs singuliers (individu, nation ou Etat) et nécessairement autour d'un secteur unique (politico-militaire, sociétal, environnemental ou économique) 27. Il requiert à cet égard la construction d'un cadre pour chaque secteur et/ou acteur choisi. Il se limite donc à l'étude isolée d'une forme précise d'interaction pour y déceler le maillage des dynamiques d'insécurité. Si cette construction présente l'attrait méthodologique de comprendre de façon approfondie un secteur, elle pose toutefois la question tout aussi cruciale, bien qu'ad quem, de leur réajustement, afin de donner une image globale des réseaux d'(in)sécurité qui tissent un complexe. Le complexe hétérogène s'écarte, en revanche, de ce monisme. Il désigne une approche pour laquelle la logique régionale de sécurité peut intégrer plusieurs types d'acteurs (Etats + nations + ONG + organisations internationales) interagissant à travers plusieurs secteurs ou enjeux (économiques + sociétaux + environnementaux + politico-militaires).

Au sens strict, peu de complexes de sécurité peuvent être proclamés homogènes. En réalité, ils sont souvent hétérogènes, tant du point de vue des acteurs que sous l'angle des secteurs d'interaction pertinents. Il s'ensuit que le choix d'un cadre analytique, de surcroît homogène, est une violence faite à la réalité sociale, un réductionnisme en somme. A contrario, si l'on déclare que les complexes de sécurité sont souvent hétérogènes, c'est que seule l'ontologie

25. Voir Wrever O., «Security, the speech act. Analyzing the politics of a word », Working Papers $\mathrm{n}^{\circ} 19$, Copenhague, Centre for Peace and Conflict Research, 1989, p. 5-6; Wrever O., "Securitization and desecuritization", in Lipschutz R.D. (ed.), On Security, New York, Columbia University Press, 1995, pp. 46-86.

26. Buzan B., op. cit.

27. Voir Nierop T., Systems and Regions in Global Politics: An Empirical Study of Diplomacy, International Organization and Trade, 1950-1991, Chichester, John Wiley \& Sons, 1994, pp. 27-29. 
relationnelle change à travers le spectre de la sécurité régionale ${ }^{28}$. La dynamique d'un complexe de sécurité est donc déterminée par l'intensité des relations amicales ou inamicales entre acteurs.

Mais ce tropisme en faveur du rapport ami-ennemi, essence structurante du complexe de sécurité, n'en demeure pas moins problématique. D'une part, parce qu'il est dépourvu d'arguments lorsqu'émerge un complexe de sécurité articulé autour de principes autres que le couple ami-ennemi. D'autre part, il pose problème parce ce que le couple conceptuel ami-ennemi, que Julien Freund qualifiait d' 'essence du politique 29 », ne rend pas suffisamment compte de la complexité des rapports qu'une entité politique entretient avec d'autres. Or, pour détecter un complexe de sécurité, il suffit qu'existe une certaine communauté d'externalités de sécurité. Mais, en la matière, le vocabulaire est incertain. Il faut donc commencer par clarifier l'usage que nous faisons du terme « externalité ». Dans cet article, l'externalité renvoie « aux coûts - externalités négatives - et aux bénéfices - externalités positives - qui n'affectent pas ou ne profitent pas uniquement à l'acteur d'où ils s'originent 30 ». En revanche, on ne parle plus d'externalité dès qu'il y a action délibérée d'un acteur pour nuire au voisin; le terme d'agression sera plus approprié. Il est frappant enfin de constater que l'externalité est aussi appelée «effet de voisinage 31 ».

En vérité, selon David Lake, «les externalités transfrontalières affectent, par définition, plusieurs Etats et sont, à ce titre, intrinsèquement politiques et stratégiques 32 ». De fait, partout où émergent des complexes de sécurité, partout où la contiguiité géographique est le véhicule des externalités, la probabilité qu'un acteur se trouve coincé entre plusieurs complexes de sécurité s'accroît. Il faut remarquer enfin que ce sont la source et la concentration des externalités qui déterminent la cible et le contenu des politiques. Ainsi, pour une communauté de sécurité pluraliste, les points de tension se trouvent le plus souvent aux frontières externes. On comprend dans ces conditions l'importance de la frontière dans le dispositif sécuritaire de l'Union :

28. Le continuum qui en résulte peut prendre trois degrés, du négatif (ennemi) au positif (ami): 1) le conflit pur se décide et se structure autour d'une interdépendance de peurs et de perceptions de menaces intersubjectives. Les cas sont nombreux : la Tchétchénie, le conflit israélo-palestinien ou la guerre des Grands Lacs en Afrique centrale ; 2) le régime de sécurité dans lequel, bien qu'il y subsiste une certaine méfiance entre acteurs, ces derniers ont adopté des mesures de réassurance permettant de contrôler le dilemme de la sécurité. A divers degrés certes, deux cas semblent pertinents : le Conseil de coopération du Golfe et la SADC ; 3) la communauté de sécurité pluraliste qui se réfère à une institutionnalisation pacifique des rapports de puissance. La guerre n'y est donc plus une option centrale. L'UE en est le cas le plus manifeste.Voir Adler E., Barnett M. (eds.), Security Community, Cambridge, Cambridge University Press, 1998.

29. Freund J., L'Essence du politique, Paris, Sirey, 1965.

30. Lake D., "Regional security complexes: a system approach ", in Lake D., Morgan P.M. (eds.), Regional Security Orders: Building Security in a New World, University Park, The Pennsylvania State University Press, 1997, p. 49.

31. Ibid.

32. Lake D., op. cit., p. 51. 
« Même à l'ère de la mondialisation, la géographie garde toute son importance. Il est de l'intérêt de l'Europe que les pays situés à ses frontières soient bien gouvernés. Les voisins engagés dans les conflits violents, les Etats faibles où la criminalité se répand, les sociétés défaillantes ou une croissance démographique explosive aux frontières de l'Europe constituent pour elle autant de problèmes 33 ».

Aussi, pour réguler ces externalités sécuritaires, l'UE met en œuvre un type particulier de gouvernance dite « externe », qui est en réalité une combinaison plus ou moins cohérente, mais intuitivement efficace, de la socialisation et de la conditionnalité ${ }^{34}$.

La section suivante montrera néanmoins qu'en l'absence de perspective d'adhésion, la gouvernance externe débouche sur un complexe de sécurité à géométrie variable, une "hiérarchie stratifiée 35 ». De plus, parce que la gestion des externalités négatives de sécurité met au contact des entités politiques de puissance inégale, aux attentes divergentes, ce complexe de sécurité conduit à un régionalisme asymétrique. N'est-ce pas l'UE qui décide de ce que sont les «valeurs communes»? N'est-ce pas elle qui juge de la qualité des efforts consentis par le voisin pour les adopter ? Plus fondamentalement enfin, n'est-ce pas l'UE qui décide du moment opportun pour passer à une forme renforcée de coopération ? Cette asymétrie est donc de principe, et non d'initiative. En effet, les partenaires souhaitent bénéficier, quoique de manière et pour des raisons différentes, des programmes de coopération offerts par l'UE, dans des secteurs spécifiques. Par exemple, c'est à la demande conjointe des présidents ukrainien (Iouchtchenko) et moldave (Voronin) que l'UE a mis sur pied une mission d'assistance à la frontière entre les deux pays. Le but principal de cette mission est, affirme Le Rapport de suivi PEV, $\mathrm{d}$ ' " améliorer les capacités des services ukrainiens et moldaves à cette frontières et d'apporter ainsi une contribution précieuse au règlement $d u$ conflit transnistrien 36 ». Toutefois, quel que soit le degré de cette liberté d'initiative, les partenaires ont en réalité très peu de contrôle, si ce n'est aucun, sur les contours et la dynamique des accords.

Ces points admis, les thèmes consubstantiellement liés que l'on examinera ci-dessous - frontières, gouvernance externe et complexe de sécurité -

33. Stratégie européenne de sécurité, Bruxelles, 12 décembre 2003, p. 7. Document disponible sur : http://www.consilium.europa.eu/uedocs/cmsUpload/031208ESSIIFR.pdf

34 . Pour une introduction à la gouvernance externe, voir Laxenex S., «EU external governance in "Wider Europe" ", Journal of European Public Policy, vol. 11, n², 2004, pp. 680-700.

35. Sur ce terme, voir Aron R., Etudes sociologiques, Paris, PUF, 1988, p. 18.

36. Commission des Communautés européennes, Document de travail des services de la Commission accompagnant la «Communication from the Commission to the Council and the European Parliament on Strengthening the European Neighbourhood Policy ", Rapport de suivi Ukraine, COM (2006) 726, p. 6. 
ont trait aux processus décisifs qui orientent notre démarche : en quoi la PEV est-elle un complexe de sécurité et en quoi peut-on dire qu'elle institue une «biérarchie stratifiée »?

\section{Géométrie variable et complexe de sécurité}

On a suggéré que le domaine crucial de la $\mathrm{PEV}$, là où l'on peut le mieux détecter son fonctionnement et ses effets, est celui de la frontière. Or, l'examen des complexes de sécurité nous l'a appris, la frontière est le point où se concentrent les tensions générées par les externalités de sécurité négative. On touche là bien entendu à la question délicate de la formation d'un complexe de sécurité et des solutions préconisées pour le réguler. Décrire cette construction revient d'abord à examiner les ressorts géographiques de la PEV. On tentera ensuite de montrer que les instruments de gestion de la frontière, sans promesse d'adhésion, entraînent deux conséquences. D’une part, dans la mesure où certains partenaires jugent insuffisante toute incitation autre que l'adhésion, le curseur de l'interaction penche vers un régionalisme asymétrique, un seul des partenaires semblant pleinement satisfait des termes de l'accord. D'autre part, tant la diversité des partenaires, que - ce qui en est en grande partie la conséquence - la divergence des attentes produisent un complexe de sécurité à géométrie variable.

La thèse centrale de cette partie est donc que les instruments qui auraient servi à lever les barrières de l'adhésion - conditionnalité et socialisation - peuvent, dans le cadre de la PEV, s'ils ne sont pas accompagnés de pédagogie, de contrôle strict et d'incitations suffisamment fortes, devenir des écueils majeurs.

\section{Frontiérisation, externalités, différenciation}

L'étude des frontières comporte généralement deux aspects qui parfois s'enchevêtrent : quel est le sens de la frontière ? Que délimite-t-elle, selon quelles modalités 37 ? Quand il s'agit de la frontière de l'UE, c'est la dernière interrogation qui devient évidemment la plus décisive, d'autres venant la compléter. Mais la gestion des frontières externes de l'UE est d'une complexité peu commune. Cette politique est en effet marquée en premier lieu, pour reprendre les termes de Berg et Ehin, par «une forte fragmentation politique, un polycentrisme institutionnel et une coordination inadéquate entre les différents piliers 38 ». En second lieu, et ce n'est pas le moins important, les politiques dont le but initial est d'assurer le fonctionnement du régime de gestion des frontières européennes, s'inspirent de paradigmes politiques et de cadres

37. Voir Postel-Vinay K., "Géopolitique et pouvoir », Critique internationale, n¹0, 2001, p. 52.

38. Berg E., Ehin P., "What kind of border regime is in the making? Towards a differentiated and uneven border strategy ", Cooperation and Conflict: Journal of the Nordic International Studies Association, vol. 41, n¹, 2006, p. 56. 
conceptuels distincts ${ }^{39}$. C'est redire, sous une autre forme, que la gestion des frontières est une "politique composite 40 ", un cadre cognitif et normatif aux contours mobiles, qui tire sa substance et sa spécificité de discours politiques concurrents, élaborés dans des espaces politiques épars. Ainsi, la gestion des frontières de l'Union est-elle le produit de trois paradigmes politiques, imprégnés d'idées, de préférences et de perceptions spécifiques sur la fonction, le mode de gouvernance et le degré d'ouverture de la frontière, à savoir : le régime de Schengen, la coopération transfrontalière sous l'égide de la coopération économique régionale et la PEV. Qu'on ne s'y trompe pas : le caractère fragmenté de cette politique n'implique pas l'inexistence de transactions entre les paradigmes, bien que ce soit là une des sources supplémentaires de l'extraordinaire enchevêtrement institutionnel du modèle européen de la gestion des frontières externes. Dans une telle perspective, la gestion intégrée des frontières, qui marque l'évolution actuelle des frontières externes de l'Union, doit alors être conçue comme une dynamique distincte dont l'apport essentiel est de subsumer les deux premiers modèles - Schengen et la coopération économique régionale - à la PEV ${ }^{41}$. C'est que, contrairement à ceux-là, la PEV infléchit les règles de Schengen, en échange d'un ordonnancement des développements sociaux, politiques et économiques des Etats voisins. Cette perspective, assez inhabituelle sans doute dans la gestion des frontières, s'appréhende comme une tendance à l'émergence d'une interdépendance stratégique entre acteurs régionaux, cloisonnés jusque là en des sites différents. Ce que l'on peut formuler autrement en tant que passage d'une forme rigide, surtout géographique, de la frontière, à une forme plus flexible qui comporte, en sus de sa fonction de séparation, une fonction de transformation et d'assimilation du voisin.

C'est en cela que réside l'intérêt, pour l'analyse de la PEV, des travaux de la géographie (critique). La production de la politique internationale est,

39. Ibid.

40. Sedelmeier U., "Sectoral dynamics of EU enlargement: advocacy, access and alliances in a composite policy ", Journal of European Public Policy, vol. 9, n4, 2002, pp. 627-749.

41 . Le concept de gestion intégrée des frontières a été développé en grande partie à la suite des programmes PHARE, TACIS, CARDS. PHARE (Poland and Hungary Assistance for the Restructuring Economy) est l'instrument financier de l'UE mis en place en 1989 pour assister les PECO (Pays d'Europe centrale et orientale) dans leur transition vers le régime démocratique et l'économie de marché. Le programme communautaire TACIS (2000-2006) encourage la démocratisation, l'Etat de droit et la transition vers l'économie de marché des nouveaux Etats indépendants (NEI), nés de l'éclatement de l'URSS, par la fourniture d'une assistance technique, de transfert de savoir-faire au secteur public et privé ou le co-financement de projets. A partir de 2007, l'aide à la plupart des pays des NEI se déploiera dans le cadre de la politique de voisinage. CARDS : Community Assistance for Reconstruction, Development and Stabilisation (Programme communautaire à la reconstruction, au développement et à la stabilisation des Balkans occidentaux). La gestion intégrée des frontières vise une harmonisation à la fois légale (mise en place d'un code des frontières Schengen), financière (partage du fardeau matériel) et opérationnelle (création de l'Agence européenne des frontières) des pratiques de gestion des frontières. Conseil de l'Union européenne, doc. ${ }^{\circ} 10019 / 02$ FRONT $58 \mathrm{du}$ 14 juin 2002 ; European Agency for the Management of Operational Cooperation at the External Borders of the Member States of the European Union, EC n²007/2004 du 26 octobre 2004 (JO L 349/204). 
comme l'affirment John Agnew et Stuart Corbridge, l'expression d'interprétations géographiques plus ou moins sédimentées ${ }^{42}$. Peu importe finalement la stratégie choisie. Il s'agit avant tout de ne pas succomber à la description géographique de la politique internationale. En revanche ce qui à cet égard nous parait fondamental, c'est, pour reprendre les termes de la géographie critique, la manière dont les acteurs mobilisent les codes géographiques, à partir d'une cristallisation des enjeux de pouvoir ou, sous certains aspects, à partir de la création d'un espace d'allégeance et de solidarité ${ }^{43}$. Dans cette perspective, tout se passe donc comme si « l'invention géographique 44 » était l'instrument des communautés politiques, pour énoncer leurs rapports au reste du monde. De plus, selon la formule de Gearoid O’Tuathail, la géographie se double d'une fonction identitaire, en ce qu'elle renvoie à la construction de l'espace politique mondial, et non seulement à un donné plus ou moins apprivoisé 45 . L'institution de la frontière a donc, en son expression épurée, une double extension : matérielle par sa fonction de séparation et cognitive par sa fonction de différenciation. Les deux extensions-fonctions sont inscrites de manière identique dans les aspirations, les objectifs et les ambitions de la PEV.

Le problème de la frontière relève principalement de la distinction structurante - que R.B.J. Walker développe sans nous en donner une présentation autre que postmoderne 46 - entre interne et externe. On y discerne, résumée de façon drastique, la question suivante : comment réguler les effets indirects (externalités de sécurité négatives) de l'intégration européenne, sans s'aliéner les nouveaux partenaires? La lettre conjointe de Christopher Patten et de Javier Solana propose pour l'essentiel de renforcer les relations avec les voisins afin de contenir les nouvelles menaces produites ou accentuées par le déplacement des frontières, né de l'intégration. Ils affirment :

«notre politique de voisinage a plusieurs objectifs : la stabilité, la prospérité, la règle de droit et les valeurs partagées le long de nos frontières sont toutes fondamentales pour notre propre sécurité. Un échec dans un seul de ces domaines confrontera l'Union à des externalités négatives 47 ».

42. Agnew J., Corbridge S., «The new geopolitics: the dynamics of geopolitical disorder ", in Johnston R., Taylor P. (eds.), A World in Crisis?, Oxford, Blackwell, 1989; Agnew J., Corbridge S., Mastering Space, Londres, Routledge, 1995.

43. Dalby S., "Critical geopolitics: discourse, difference and dissent ", Environment and Planning, n9, 1991 ; O’Tuathail G., Critical Geopolitics, Minnesota, University of Minnesota Press, 1996.

44 . Postel-Vinay K., « Géopolitique et pouvoir », op. cit., p. 54.

45. O’Tuathail G., op. cit.

46. Encore que certains Etats puissent se retrouver dans une zone grise, ni dedans, ni absolument dehors. Ce qui pourrait alors faire apparaitre le voisinage comme une zone-tampon, un filtre des menaces. Walker R.B.J., Inside / Outside: International Relations as Political Theory, Cambridge, Cambridge University Press, 1992.

47 . Patten C., Solana J., op. cit. 
Du point de vue de l'UE en effet, la contiguiité géographique entraîne, et c'est là un point crucial souvent négligé, une redistribution des responsabilités de part et d'autre de la ligne de démarcation. La Commission l'a bien saisi, elle qui précise: "un voisinage partagé implique, par sa nature même, un partage $d u$ fardeau et une responsabilité conjointe pour répondre aux défis (conflit et insécurité) qui menacent la stabilité 48 ».

Si nous passons maintenant du discours sur la nécessité de coopérer à l'émergence de la PEV, prise ici dans ses grandes lignes comme le résultat de compromis inter-bureaucratiques, la question de la délimitation interne/externe - apparaît plus clairement. En effet, en amont de la PEV se trouve une communication de la Commission au titre plus englobant, $L$ 'Europe élargie ${ }^{49}$. Ce projet politique reprenait de fait tant les Etats du continent européen que les Etats extra-continentaux ${ }^{50}$. Le schéma ci-contre (table 1) décrit la «biérarchie stratifiée » qui en découle.

On objectera que le nouveau titre "politique européenne de voisinage » n'est qu'un changement de label. Mais c'est là un raccourci risqué. La notion d' « Europe élargie » (ou de « Grande Europe ») a selon nous une densité symbolique dont l'UE n'a très probablement perçu la portée qu'a posteriori. Reste que l'UE semble parfois user du terme « Europe » avec une certaine restriction, comme si «Union européenne » et «Europe » renvoyaient à une réalité identique. Le document pour la Stratégie européenne de sécurité s'ouvre ainsi : "l'Europe n'a jamais été aussi prospère, aussi sûre, ni aussi libre. La violence de la première moitié du vingtième siècle a cédé la place à une période de paix et de stabilité sans précédent dans l'histoire européenne 51 ». Ce dont il s'agit, c'est bien de l'Union européenne, non pas du continent européen, dans la mesure où sécurité, liberté et prospérité y demeurent, à certains endroits, particulièrement fragiles.

La politique «européenne » de voisinage scelle la pratique, notamment parce qu'elle atteste de l'emprise de l'UE sur le terme «Europe ». De là provient l'idée couramment répandue que la $\mathrm{PEV}$, c'est la politique de voisinage de l'Union européenne. Le procédé discursif de brouillage sémantique n'est pas banal ; il dépose la marque «Europe». Sans doute serait-il déraisonnable de tout ramener au discours. Mais on aurait tort d'en minimiser la force cognitive, qui porte, en plus de la production des sujets (Europe, voisinage), sur l'établis-

48. Commission des Communautés européennes, L'Europe élargie - Voisinage : un nouvean cadre pour les relations avec nos voisins de l'Est et du Sud, COM(2003), 104, p. 12.

49. Ibid.

50. Voir Wrever O., «Europe's three empires: a watsonian interpretation of post-wall European security », in Fawn R., Larkins J. (eds), International Society after the Cold War: Anarchy and Order Reconsidered, Londres, MacMillan, 1996.

51. Stratégie européenne de sécurité, Bruxelles, 12 décembre 2003, p. 1. Document disponible sur : http://www.consilium.europa.eu/uedocs/cmsUpload/031208ESSIIFR.pdf 
sement du sens et des termes d'action ${ }^{52}$. Les catégories «Grande Europe » ou «politique européenne de voisinage » sont toutes, à des degrés divers, des instances de la puissance productive du discours, étant entendu qu'elles génèrent du sens, conditionnent les termes du débat et définissent les politiques permises, possibles, imaginables ou désirables. De sorte qu'analyser la puissance productive du discours conduit à se concentrer sur la capacité des processus sociaux à créer des sujets particuliers, à fixer le sens et à construire des catégories ${ }^{53}$. Plus particulièrement, l'abandon du groupe lexical « Europe élargie » au profit de la «politique européenne de voisinage » préfigure un changement radical de posture : alors que dans le premier cas l'UE était, pour ainsi dire, primus inter pares (premier parmi les égaux), dans le second cas, il y a une rupture nette : l'UE et les autres. La frontière est chargée d'en assurer la médiation.

Table 1. Distribution des groupes qui entourent l'Union européenne 54

\begin{tabular}{|c|c|}
\hline Groupes & Pays de l'Europe Elargic \\
\hline (iroupe n ${ }^{\prime \prime} 1$. Noyau central de l'UJ! & $\begin{array}{l}\text { Autriche, Belgique, linilande, lirance, Allemagne, } \\
\text { Grìce, Italic, Luxcmbourg, Pays-Bas, Portugal } \\
\text { lispagne }\end{array}$ \\
\hline $\begin{array}{l}\text { (iroupe n"2. Membres bénéficiant d'un } \\
\text { systíme d'intćgration flexible dit « opl- } \\
\text { out»" }\end{array}$ & I)anemark, Irlande, Suède, Royaume-Inni \\
\hline (iroupe n 3 . Nouveaux litats-membres & $\begin{array}{l}\text { (hypre, République tchèque, listonie, Ilongrie, } \\
\text { Léthnic, Lituanic, Mallic, Pologne, Slovaquic } \\
\text { Slovénie, I3ulgarie, Roumanie }\end{array}$ \\
\hline (itoupe n'4. Membres de l'lilili: & Islande, Norvège, Suisse \\
\hline $\begin{array}{l}\text { Groupc } \mathrm{n}^{0} 5 \text {. Négocialcurs, non- } \\
\text { négocialcurs cit candidats ćventucls }\end{array}$ & $\begin{array}{l}\text { Croalic, Turquic, Macédoinc, Scrbic, Montćnégro, } \\
\text { Bosnic-Herź́govinc, Abanic }\end{array}$ \\
\hline Groupe n 6. Voisitis européens & $\begin{array}{l}\text { Arménie, Azerbaidjan, lizélorussie, (ieorgie, } \\
\text { Moldavic, Russic, Ukrainc }\end{array}$ \\
\hline Groupe $n^{\circ} 7$. Voisins $n$ & $\begin{array}{l}\text { Mlgćric, Egyplc, Isrä̈l, Jordanic, Libyc, Maroc, } \\
\text { Autorité palestinienne, Syrie, 'lunisie. }\end{array}$ \\
\hline
\end{tabular}

Le processus de frontiérisation s'accompagne bien souvent d'un projet identitaire, plus ou moins explicite, qui peut soit le précéder, soit lui être parallèle, ou encore en être le résultat ${ }^{55}$. Mais quelle que soit la séquence (identité-frontière, frontière-identité), il semble pertinent de relever la portée analytique de ce lien.

52. Buzan B., Wxever O., de Wilde J., op. cit.

53. Barnett M., Duval R., "Power in international politics », International Organization, vol. $58, \mathrm{n}^{\circ} 1,2005$, pp. 39-75.

54 . Schéma revu et corrigé, extrait de Tassinari F., "Security and integration in the EU neighbourhood: the case for regionalism ", op. cit., p. 2.

55 . Lapid Y., "Identities, borders, orders: nudging international relations theory in a new direction ", in Mathias A., Jacobson D., Lapid Y. (eds.), Identities, Borders, Orders: Rethinking 
Car comprendre comment évolue et se manifeste le nœud «frontière-identité », et vice versa, permet de rompre avec l'approche rationaliste des relations internationales, qui se concentre sur les structures matérielles, dans leurs modalités et dans leurs conséquences - la pérennité de la frontalité des rapports internationaux. Dans ce cadre, tout est antérieur aux interactions et aux processus. En d'autres termes, rien ne permet de rendre compte des rapports co-constitutifs entre frontière et identité. Ainsi, lorsque l'on parle du lacis formé par l'identité et la frontière, l'apport théorique tourne, nous semble-t-il, au bénéfice du constructivisme. On devine l'intérêt que représente une telle posture pour la PEV. Quelle que soit la place que l'on y accorde au discours, la PEV a pour projet décisif la «fabrication » de nouveaux voisins ${ }^{56}$. Ces derniers ne sont pas décrits comme des ennemis, mais ils ne se confondent pas non plus avec les membres de l'UE. Leur identité est en chantier, puisque : "notre objectif ", affirme le document Stratégie européenne de sécurité, "est de promouvoir un cercle d'Etats bien gouvernés, avec lesquels nous pouvons partager des relations d'affinités et de coopération, à l'Est de l'Union européenne et aux frontières de la Méditerranée 57 ». En somme, plus on met l'accent sur la constitution des acteurs à travers des systèmes de signification et des pratiques discursives, plus le constructivisme fournit un cadre fiable - et à certains égards plus robuste que tous les « néos 58 »-pour penser la production des acteurs et des identités.

La controverse, scientifique et technique, autour du concept d'identité semble insoluble, à cause notamment de la forte charge émotionnelle dont elle se nourrit. Le débat est immense et saturé d'équivoques. Toutefois, les travaux notamment de Manuel Castells ${ }^{59}$, Julia Kristeva 60 et Henri Tajfel 61 nous permettent, eu égard à ce qui vient d'être dit, de tempérer la difficulté. En dépit de leurs différences, ces auteurs se rencontrent sur la conviction essentielle qu'un des traits spécifiques de l'identité est la catégorisation et son corollaire, l'exclusion. Or, l'ex-commissaire européen Günther Verheugen ne dit rien d'autre quand il soutient : "Je pense que quiconque croit que l'Ukraine devrait rejoindre l'Union européenne [...] devrait aussi peut-être défendre l'argument selon lequel le Mexique doit être membre des Etats-Unis 62 ». En 2000, Romano Prodi comparait la candidature de l'Ukraine à celle de la Nouvelle-Zélande. En résumé et plus abruptement, la Nouvelle-Zélande et l'Ukraine ont en partage la différence radicale avec l'UE. Ces positions révèlent que le décret discursif (par un acte à

International Relations Theory, Minneapolis, University of Minnesota Press, 2001, pp. 1-20.

56 . Voir les textes de J. Jeandesboz et R. Zaiotti dans ce numéro.

57 . Stratégie européenne de sécurité, Bruxelles, 12 décembre 2003, op. cit., p. 8.

58. C’est-à-dire essentiellement le néoréalisme et le néolibéralisme.

59. Castells M., Le Pouvoir de l'identité, Paris, Fayard, 1999.

60 . Kristeva J., Etrangers à nous-mêmes, Paris, Fayard, 1988.

61. Tajfel H., Human Groups and Social Categories: Studies in Social Psychology, Cambridge, Cambridge University Press, 1981.

62. Verheugen G., cité dans Primatorova A., «In search of two distinct tracks for non-EU Europe and the European Neighbourhood Policy », in Hayoz N., Jesien L., Van Meurs W. (eds.), Enlarged EU-Enlarged Neighbourbood, Bern, Peter Lang, 2005, p. 33. 
force illocutoire de nature politique), et non la localisation sur le continent européen, détermine l'appartenance à l'UE ${ }^{63}$.

On objectera à juste titre que la PEV en son rapport à l'identité et aux frontières, est beaucoup plus complexe que le récit que nous venons d'en livrer. La fonction de la gouvernance de la sécurité à l'Est a peu en commun avec le régime de sécurité en vigueur au Sud ${ }^{64}$. Mais cette conviction cesse d'apparaître comme une objection décisive dès lors qu'est prise en compte l'ambiguité délibérément entretenue par la Commission :

« La nouvelle politique de voisinage a pour but de définir un cadre pour le développement d'une nouvelle relation qui, à moyen terme, ne prévoirait pas la perspective d'une adhésion ou un rôle dans les institutions européennes. Les problèmes pratiques posés par la proximité et le voisinage devraient donc être traités indépendamment d'une éventuelle adhésion à l'Union ${ }^{65}$ ».

Cela suppose en d'autres termes que la promotion des réformes politiques, sociales et légales qui précèdent en principe la transformation interne des partenaires, doit être traitée comme une fin en soi. Simultanément toutefois, la PEV promet à chaque Etat une "relation privilégiée », nourrie par «l'attachement partagé aux valeurs communes $\gg{ }^{66}$. En vérité, le degré de souscription aux valeurs communes conditionne la qualité des échanges que le partenaire peut espérer de l'Union ${ }^{67}$.

Cette exigence, est-il besoin de le dire, n'est pas inédite. Elle avait déjà fortement marqué l'intégration européenne, qui combinait sous leur forme instrumentale la socialisation et la conditionnalité, bien que, dans le cas de la PEV, leur statut soit plus difficile à justifier, étant donné que la finalité - l'adhésion - est diversement accessible aux uns et aux autres. Inévitablement, les deux principes dépendent l'un de l'autre, mais ne doivent pas se confondre l'un avec l'autre. En revanche ils servent, comme on le montrera par la suite, des objectifs similaires : repousser la zone d'incertitude, définir les frontières de l'Union et établir les prémisses d'une identité européenne. Par conséquent, l'analyse des leviers de la PEV devrait avoir pour but de marquer la faisabilité et les apories de la conditionnalité et de la socialisation, quels que doivent être leurs résultats, parce que pour l'UE ces principes sont évidemment des vecteurs d'influence ou, si l'on préfère, de puissance.

63. Comparer avec le volet légal (article 49 TUE).

64. Voir Zielonka J., Europe Unbound: Enlarging and Reshaping the Boundaries of the European Union, Londres, Routledge, 2002.

65. Commission des Communautés européennes, L'Europe élargie - Voisinage : un nouvean cadre pour les relations avec nos voisins de l'est et $d u$ sud, $\operatorname{COM}(2003), 104$, op. cit.

66. Commission des Communautés européennes, Politique européenne de voisinage - Document d'orientation, COM(2004), 373.

67. Kelley J., « New wine in old wineskins: promoting political reforms through the new European Neighbourhood Policy ", Journal of Common Market Studies, vol. 4, n¹, 2006, pp. 29-55. 
L’argument primordial de la conditionnalité est formulé par la Commission :

« l'ambition et le rythme de développement des relations de l'Union européenne avec chaque pays partenaire dépendront de son engagement en faveur des valeurs communes, ainsi que de sa volonté et de sa capacité à mettre en œuvre les priorités convenues 68 ».

Ou encore, à propos de l'Ukraine : «Le niveau de l'évolution de leurs relations tiendra pleinement compte des efforts déployés par l'Ukraine et des avancées concrètes réalisées, par l'Ukraine dans le respect de ses engagements en faveur de valeurs communes 69 ». Pour être juste avec les textes communautaires, il nous faut apprécier l'évolution du discours sur la conditionnalité, parce qu'elle permet d'apprécier l'effet pédagogique de l'élargissement sur les institutions européennes, parce qu'elle permet de comprendre comment cet apprentissage a influencé la PEV. Elles y ont en effet appris qu'il valait mieux valoriser les réalisations même partielles - que d'exiger des progrès isomorphes dans tous les secteurs de coopération. Ainsi, à la différence de la communication sur l'Europe élargie 70 qui prévoyait un régime de conditionnalité strict, le document stratégique de la PEV adopte un vocabulaire moins abrupt, parce qu'il rappelle à plusieurs reprises ce que la PEV permet, plutôt que les contraintes qu'elle charrie. On aurait tort d'en conclure, cependant, que la conditionnalité occupe une place moins importante; un discours plus sensible aux crispations des partenaires ne se traduit pas nécessairement par une posture politique moins exigeante.

Bien entendu, le succès de la conditionnalité dépend de sa crédibilité, qui est tributaire de l'attrait que représente l'incitation qu'offre l'Union à ses partenaires ${ }^{71}$. Cette incitation doit en outre faire sens pour les acteurs, parce qu'elle structure leurs perceptions, leurs interprétations et constitue l'armature de leurs calculs et de leurs actions. La PEV en propose plusieurs, de nature économique, politique et sociale. On peut citer, sans prétendre en épuiser la liste : (a) la perspective d'aller au-delà de la coopération, vers une intégration renfor-

68. Commission des Communautés européennes, Politique européenne de voisinage-Document d'orientation, op. cit., p. 8.

69. Commission des Communautés européennes, Proposition de décision du Conseil relative à la position à adopter par les Communautés et leurs Etats-membres au sein du Conseil de coopération institué par l'accord de partenariat et de coopération établissant un partenariat entre les Communautés européennes et leurs Etats-membres, d'une part, et l'Ukraine, d'autre part, en ce qui concerne l'adoption d'une recommandation portant sur la mise en ouvre du plan d'action UE-Ukraine, COM(784), p. 8. Voir aussi Ferrero-Waldner B., Press conference to launch first seven Action Plan under the European Neighbourhood Policy, 9 décembre 2004.

70. Commission des Communautés européennes, L'Europe élargie - Voisinage : un nouvean cadre pour les relations avec nos voisins de l'Est et du Sud, op. cit.

71. Smith K., "The use of political conditionality in the EU's relations with third countries: how effective? ", European Foreign Affairs Review, vol. 3, n², 1998, pp. 253-274. 
cée (par exemple, à travers la participation au marché intérieur de l’Union) ; (b) la réduction des barrières commerciales et l'ouverture des économies; (c) l'augmentation du soutien financier; (d) la participation aux programmes de la Communauté, notamment : la promotion des échanges culturels, techniques et scientifiques; (e) le soutien à l'harmonisation législative en vue de s'aligner sur les exigences de l'UE. Aux Etats les plus studieux, l'UE assure plus : une coopération administrative renforcée, un dialogue ouvert sur la politique de visa. C'est cette promesse, ou plus exactement ce dont elle augure, qui explique que l'Ukraine et la Moldavie par exemple, militent pour une relation contractuelle consolidée. L’UE est toutefois formelle : le problème des visas

« ne peut, bien entendu, être réglé que dans le cadre d'un examen plus vaste consacré à des questions telles que la coopération en matière d'immigration clandestine [...] la lutte contre la traite et le trafic illicite des êtres humains, l'efficacité de la gestion des frontières, les accords de réadmission [...] $]^{72}$ ».

Les voisins de l'UE deviennent à leur tour les porteurs de nouvelles exigences vis-à-vis de leurs voisins. Une façon pour l'Europe de gérer les rapports avec les «voisins de ses voisins ${ }^{73}$ ». L'Ukraine a donc, pour satisfaire l'UE, signé une douzaine d'accords bilatéraux de réadmission. L'engouement de l'Ukraine s'explique assez facilement. Pour elle, en effet, toute coopération renforcée avec l'UE peut être le premier pas vers des négociations d'adhésion. Sur ce point cependant, l'UE est restée réservée, même si là aussi sa position a évolué, passant d'un credo peu flexible - « tout sauf les institutions » 74 - à un discours tour à tour agnostique - « la PEV ne prépare pas à l'adhésion, mais elle ne l'exclut pas non plus »et plus pragmatique - « on verra au cas par cas » 75 .

Ceci dit, l'existence admise de cette ambiguïté suffit à montrer que le seul vecteur de la conditionnalité ne permet pas à l'UE, en l'absence de la perspective d'adhésion, de susciter suffisamment de ralliement à sa politique de voisinage chez certains de ces voisins stratégiques. La Libye considère par exemple, au vu de ce qui est offert, que la PEV n'est pas une priorité ${ }^{76}$. Par conséquent, l'UE développe en parallèle une « diplomatie douce » dite plus précisément de «socialisation », dont le but n'est pas d'imposer mais de transmettre aux acteurs des matrices cognitives à travers lesquelles s'expriment les objec-

72. Commission des Communauté européennes, Communication de la Commission au Conseil et au Parlement relative au renforcement de la politique européenne de voisinage, $\operatorname{COM}(2006), 726$, p. 6.

73 . Ibid., p. 13.

74. Prodi R., op. cit.

75. Verheugen G., The European Neighbourbood Policy, Prime Ministerial Conference of the Vilnius and Visegrad Democracies: "Towards a Wider Europe: The New Agenda, Bratislava », 19 mars 2004, SPEECH/O4/141.

76 . Interview d'un officiel libyen, Bruxelles, octobre 2006. 
tifs politiques de l'Union ${ }^{77}$. Persuadés du contenu bénéfique de ces matrices, les acteurs du voisinage devraient, la force de la pratique aidant, se réapproprier les principes politiques, les normes économiques et les représentations du monde propres à l'UE 78 .

La littérature sur la socialisation présente une telle diversité qu'il serait présomptueux de prétendre donner une représentation fournie de toutes ses subtilités en quelques lignes. On peut dépasser une telle difficulté de deux manières : d'une part, en renvoyant à l'abondante littérature néolibérale et constructiviste des relations internationales sur le sujet 79 . D'autre part, en lui donnant un contenu précis, c'est-à-dire limité à la tâche qui nous occupe. Il y a donc socialisation lorsqu'un acteur, individuel ou collectif, induit, par pression sur la réputation et à travers la persuasion, un changement comportemental ${ }^{80}$. Or dans la plupart des programmes de coopération extérieure de l'UE, on l'a dit, la socialisation tente de répondre à une fonction essentielle: exporter ses normes et valeurs. Tel est en substance le message de Romano Prodi : «le but [de la PEV] est de greffer dans ces régions un ensemble de principes, valeurs et normes qui définissent l'essence même de l'Union européenne 81 ». Durant l'élargissement, le medium de l'apprentissage a pris la forme de voyages fréquents d'officiels européens auprès des pays candidats afin de négocier, mais aussi de stimuler, le débat interne sur des questions comme la démocratie, la défense des droits de l'Homme et la reconnaissance des droits des minorités. Le contenu de la stratégie de socialisation de la politique de voisinage est calqué sur ce modèle.

Cependant, il n’y a pas de contenu politique viable sans méthode. Dès lors, pour éviter que la promotion des valeurs ne soit mal perçue et mal reçue, l'Union a forgé le concept d' " appropriation commune ", qui doit favoriser l'engagement moins réservé des partenaires à la négociation de priorités et à l'élaboration des plans d'action. Mais l'appropriation commune revêt diverses modalités. En plus des interactions fréquentes entre les acteurs officiels, l'Union accorde un rôle important à la transformation de la société civile, qui est censée être une contreforce plus ouverte au changement, capable de mobiliser à un niveau de réalité différent la substance des plans d'action pour faire, le cas échéant, pression sur le

77 . Muller, P., Les Politiques publiques, Paris, PUF, 2003, chap. 3.

78 . Petiteville F., "Exporting "values”?: EU Eexternal cooperation as "soft diplomacy” ", in Knodt M., Princen S., Haukkala H. (eds.), Understanding European Foreign Relations, Londres, Routledge, 2003, p. 123.

79. Pour une introduction très instructive à cette problématique, voir Breslauer G, Tetlock P. (eds.), Learning in US and Foreign Policy, Boulder, Westview Press, 1991; Soest D., Ruediger W., "Institutional learning within the WTO regime ", International Journal of Sustainable Development, vol. 4, n³, 2001, pp. 304-320; Stein J., « Political learning by doing: gorbachev as uncommitted thinker and motivated learner ", International Organization, vol. 48, n², 1994, pp. 155-183.

80 . Johnston I, "Treating international institutions as social environments ", International Studies Quarterly, vol. 45, n 4, 2001, p. 488.

81. Prodi R., A Wider Europe - A Proximity Policy as the Key to Stability, op. cit. 
gouvernement. Mais on aurait tort de penser que la société civile est, en dehors de l'Etat, la seule cible de la politique d'influence de l'UE. L'existence d'une telle société, qui doit souvent s'exposer à des représailles, supposerait dans bien des cas que certains principes démocratiques aient été intériorisés par des pans suffisamment représentatifs de la société. Du coup, l'UE se préoccupe de susciter l'adhésion d'autres segments de la société - les églises, les militaires ou encore les (ex-) familles royales - là où subsiste une lignée relativement influente.

L'appropriation commune s'accompagne d'une seconde technique, qui se situe en amont et en aval d'elle : la méthode ouverte de coordination qui fixe les objectifs à atteindre, mobilise le benchmarking, établit un calendrier, c'est-à-dire le rythme des réformes, et enfin procède à une évaluation des progrès accomplis, conformément aux indicateurs définis a priori. On détecte en creux une expression supplémentaire de l'influence sociale de l'UE. De fait, la méthode ouverte de coordination vise aussi à attribuer officiellement des bons et des mauvais points aux voisins qui, valorisés ou humiliés, pense-t-on, seraient enrôlés de facto dans une émulation permanente. Quel que soit le mérite ou le démérite de l'instrument, l'effet recherché est, à travers la promesse d'une coopération renforcée, d'adopter les modèles économiques, sociaux et politiques de l'UE ${ }^{82}$. C'est probablement sous cet angle qu'il serait le plus rigoureux d'envisager la gouvernance externe, laquelle voudrait capturer en une seule expression les métamorphoses de l'action de l'Union, à l'interface de l'interne et de l'externe.

La gouvernance externe, on l'oublie trop souvent, ce n'est pas la fin de l'Europe puissance civile - quel que soit au demeurant le caractère incertain de ce terme - mais la substitution d'un unique schéma d'action politique, avec ce que cela comporte d'impasses, aux nombreux instruments d'influence de l'UE. Il n'est pas indifférent pour la compréhension de la PEV que ce type de modèle trouve un large écho auprès des chercheurs dont l'Europe est le terrain de travail ${ }^{83}$. Mais ce concept ne doit pas masquer l'essentiel. Il ne suffit pas, nous semble-t-il, pour être innovant, de substituer aux termes traditionnels de conditionnalité et de socialisation une autre illusion conceptuelle. En d'autres termes, et la suite permettra de le comprendre, le concept de gouvernance externe est d'une utilité singulièrement marginale, s'il se cantonne comme c'est parfois le cas à la possibilité qu'il nous offre de nommer autrement la socialisation subie par les partenaires de la PEV. En revanche, pour autant qu'elle permette de saisir les dynamiques distinctes à l'œuvre au cœur

82 . Kelley J., «International actors on the domestic scene: membership conditionality and socialization by international institutions ", International Organization, vol. 58, ${ }^{\circ} 3,2004$, pp. $425-458$.

83. Voir entre autres Laxenex S., «EU external governance in "Wider Europe" », Journal of European Public Policy, vol.11, $\mathrm{n}^{\circ} 4$, 2004, op. cit., pp. 680-700; Schimmelfennig F., Sedelmeier U., " Governance by conditionality: EU rule transfer to the candidate countries in Central and Eastern Europe ", Journal of European Public Policy, vol. 11, n4, 2004, pp. 661-679. 
de la PEV, la gouvernance externe peut présenter un certain intérêt. C'est donc assez paradoxalement par ses carences autant que par ses intuitions que ce dispositif théorique peut nous permettre d'affiner notre propre perspective.

Pour aller à l'essentiel, c'est l'extension d'une partie de l'acquis communautaire (et on gagne à réserver l'usage à ce cadre) à des Etats non membres qui fonde le principe de la gouvernance externe. Ainsi compris, l'intérêt de la gouvernance externe pour notre propos tient en quelque sorte à son rapport à la frontière. Tout d'abord, le but de la gouvernance externe est de découpler la frontière géographique de ce que Michael Smith appelle la "frontière légale ", c'est-à-dire l'espace au sein duquel s'applique les principes du droit communautaire ${ }^{84}$. En d'autres termes, et c'est là le point où il nous importe de parvenir, la gouvernance externe cherche essentiellement à :

« internaliser les relations entre les Etats, celles des Etats-membres autant que celles avec les Etats hors Union européenne. Cela veut dire essayer d'injecter aux problèmes internationaux le sens de la responsabilité commune et les structures de la politique contractuelle naguère associée exclusivement aux affaires intérieures, et non aux affaires internationales, c'est-à-dire étrangères 85 ».

Elle combine dès lors une stratégie de politique étrangère tendue vers la stabilisation et l'adhésion, et la volonté d'associer, à travers la méthode ouverte de coordination, les voisins à la réalisation d'objectifs de politique intérieure, comme cela fut le cas avec le transfert de l'acquis communautaire aux Etats, alors candidats à l'adhésion, d'Europe centrale et d'Europe de l'Est. Mais à dire vrai, cet objectif rencontre des difficultés immenses, dans le cadre de la PEV. En effet, la méthode ouverte de coordination, avec son régime de conditionnalité, ne peut provoquer d'émulation productive que si l'incitation est à la hauteur des espérances des partenaires. Mais en va-t-il ainsi ? L'analyse des plans d'action et de nombreuses enquêtes de terrain semblent indiquer que, faute de perspective d'adhésion, la PEV ne présente qu'un attrait limité pour une partie importante des partenaires ${ }^{86}$. C'est dire qu'il existe un décalage réel entre ce que l'UE peut ou veut céder et ce que les voisins pensent pouvoir en tirer. Ce

84 . Voir Smith M. «The European Union and a changing Europe: establishing the boundaries of order ", Journal of Common Market Studies, vol. 34, n¹, 1996, p. 15.

85 . Duchêne F., "The European community and the uncertainties of interdependence ", in Kohnstamm M., Hager W. (eds.), A Nation Writ Large? Foreign Policy Problems before the European Community, Londres, Macmillan, 1973, pp. 19-20.

86. L'auteur a réalisé de nombreuses enquêtes de terrain entre septembre 2005 et novembre 2006, notamment auprès des autorités ukrainiennes, moldaves, tunisiennes et marocaines. Ce texte en est bien sûr imprégné. Toutefois, parce qu'il porte essentiellement sur les logiques et effets générés par la PEV telle que construite par l'UE, il ne reprend pas de manière systématique le contenu des discussions avec les différents fonctionnaires impliqués dans la mise en œuvre de la PEV, au sein des pays partenaires. Une telle lecture de la PEV, de « l'autre côté du miroir », pour ainsi dire, devrait constituer l'ossature d'un travail plus large, en préparation. 
décalage tient à deux causes principales : d'une part, la difficulté de maintenir un équilibre entre adhésion et partenariat, d'autre part, la difficulté de concilier une paire de principes a priori contradictoire, la différenciation et le holisme. Tout cela débouche non pas sur un mais sur plusieurs complexes de sécurité. Ceux-ci tendent corrélativement vers une PEV à géométrie variable. Plus précisément, le concept de "géométrie variable » veut à la fois souligner la diversité des externalités de sécurité et signifier que la gestion des externalités varie, en intensité et en substance, d'une frontière à l'autre de l'UE. Un schéma permet de visualiser le complexe de sécurité qui en résulte .

Figure 1. Cartographie d'un complexe de sécurité à géométrie variable 87

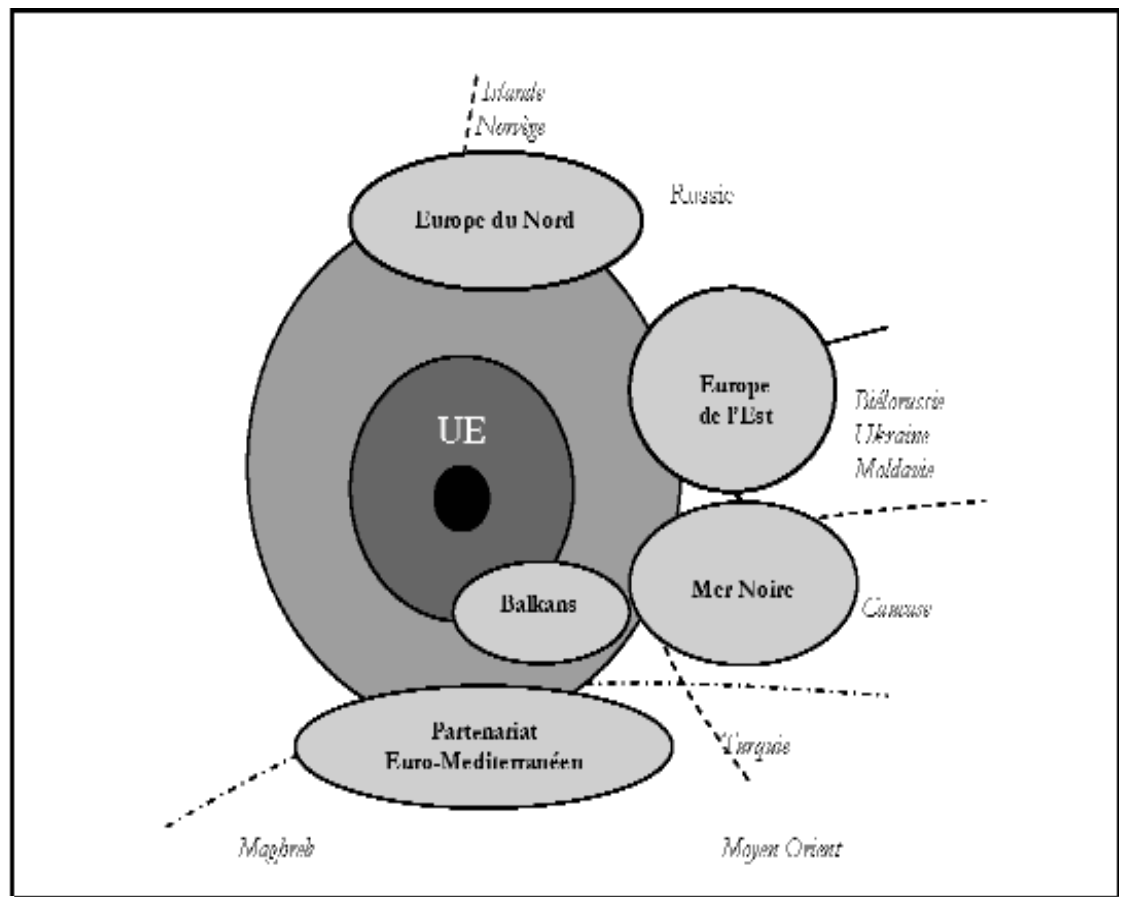

Il n'est pas besoin d'analyser toutes les configurations que revêtent ces régions, d'autant plus que seuls les Etats du Caucase, de la Méditerranée et de l'Europe de l'Est font partie de la PEV ${ }^{88}$. Il n'est pas davantage possible, dans les limites de cet article, de fournir une lecture détaillée des trois complexes pertinents de sécurité. En vérité, chaque partenaire, enchâssé dans une région, mérite-

87. Ce schéma est tiré de Tassinari F., "Security and integration in the EU neighbourhood: the case for regionalism », CEPS Working Document, n²26, juillet 2005, p. 12. Bien sûr l'exercice a ses limites, dans la mesure où en Europe, comme ailleurs, les régions se chevauchent et changent de contours en fonction de la manière dont sont exprimées les identités, de la manière dont sont articulés les intérêts et des variations des circonstances géopolitiques.

88. En outre, les Balkans ne font pas partie de la PEV parce que ces derniers ont une réelle perspective d'intégration. Voir Bosse G., "Values in the EU's neighbourhood policy: political rhetoric or reflection of a coherent policy? ", European Political Economy Review, n7, 2007, pp. 38-62. 
rait dans un travail qui aurait pour niveau d'analyse le bilatéral un examen singulier. Toutefois, au-delà de ce niveau d'analyse, est-il possible de distinguer les traits saillants des trois complexes susmentionnés ? Cette tâche n'est peut-être pas, en tant que telle, plus fragile que la précédente, même si elle est davantage menacée par la dispersion. L'étude des complexes de sécurité nous apporte non pas le contenu des relations au détail près mais une sorte de schème qui permet de déceler les stratégies (inégales) que l'UE adopte à l'égard des différentes régions qui composent la PEV.

\section{La région méditerranéenne}

L'UE y a joué un rôle particulièrement important dans l'établissement d'une structure régionale avec laquelle, et au sein de laquelle, peuvent s'instaurer des rapports multilatéraux. La coopération y est en effet organisée autour de quatre axes, social, économique, culturel et humanitaire ${ }^{89}$. La structure de la PEV est fortement modelée sur ces canevas . Ici, l'aspect économique s'est développé à un rythme régulier, presque sans entraves. Le volet énergétique, dans le cas du Maroc, par exemple, semble évoluer dans le sens souhaité par l'UE qui, à l'occasion du Rapport de suivi 2006, souligne les efforts consentis par le Royaume :

« Le Maroc joue un rôle dans la sécurité énergétique de l'UE notamment parce qu'il fait transiter du gaz vers la péninsule ibérique et qu’il exporte de l'électricité vers l'Espagne. [...] Le code des hydrocarbures rénové a permis de dynamiser les activités d'exploration 90 ».

Cependant, en dépit de la rhétorique sécuritaire (immigration, terrorisme, trafic de drogue) et de la primauté accordée dans les textes au respect des droits de l'Homme, à la promotion de la démocratie et de la règle de droit, peu de résultats notables sont à créditer à l'action de l'UE. On peut néanmoins relever quelques changements sous l'incitation de l'UE, notamment la réforme du code de la famille au Maroc, qui accorde aux femmes des droits inédits. Pour le reste, l'Egypte de Mubarak est toujours gouvernée par une loi d'exception permanente, l'activité des ONG est sous contrôle et les mouvements d'opposition disposent de peu de marge de manœuvre, quand l'accès aux élections ne leur est pas purement proscrit. Quant à la Syrie, l'Algérie, la Jordanie et la Tunisie, elles sont tout simplement loin de remplir le cahier des charges imposé par les plans d'action. Enfin, un des protagonistes les plus sérieux de la sécurité régionale, la Libye, pays de transit d'une des externalités les plus redoutées par l'Union (l'immigration illégale), reste peu intéressée par la PEV.

89. Elle a été surtout portée par le prgramme MEDA.

90. Commission des Communautés européennes, Document de travail des services de la Commission accompagnant la «Communication from the Commission to the Council and the European Parliament on Strengthening the European Neighbourhood Policy ", Rapport de suivi Maroc, $\operatorname{COM}(2006)$ 726, p. 12. 
En vérité, comme le montre J. Kelley, la PEV doit surmonter deux problèmes majeurs : les amitiés des chefs d'Etats et le syndrome algérien ${ }^{91}$. Le premier, le plus regrettable, est l'attachement personnel de certains chefs d'Etats européens à certains dirigeants de la région méditerranéenne. Un officiel de la Commission le déplore: "les éléments fondamentaux des droits de l'Homme créent des attentes. Et Chirac affiche sa sympathie à l'égard de Ben Ali 92 ». Le second facteur de régression éventuelle (dans le sens du statu quo), est le dilemme entre la stabilité à court terme et l'amélioration de la démocratie dans le long terme. En effet, l'UE redoute que les efforts économiques et sociaux menant à un système démocratique n'ouvrent une fenêtre d'opportunité 93 favorable aux partis islamistes, lesquels pourraient radicaliser leur politique étrangère ou déstabiliser, par effet de domino, la région toute entière. Ce dilemme, plus encore que les divergences réelles ou supposées entre la Commission et une partie des élites des Etats-membres pourrait expliquer pourquoi en fin de compte l'UE n'a pas appliqué un régime strict de conditionnalité à la région méditerranéenne. De fait, les critiques à l'égard d'Etats particuliers ont souvent été très allusives. Par exemple, en 2004, le rapport sur la Tunisie notait :

« La Tunisie est une république présidentielle constitutionnelle [...]. Le président actuel, S.E. Zine El Abidine Ben Ali, exerce ses fonctions depuis 1987 ; dans les dernières élections de 1999 il a recueilli 99,4\% des votes. La réforme constitutionnelle approuvée par référendum en mai 2002 a abolit la limitation du nombre de mandats présidentiels ${ }^{94}$ ».

Plus loin, pour signifier que la liberté d'association est entravée, le texte souligne: "l'installation d'une ONG étrangère est régulée par une loi spécifique. Une telle association ne peut [...] conduire ses activités en Tunisie si elle n'a pas reçu l'approbation du secrétaire d'Etat à l'Intérieur et du ministre des Affaires étrangères 95 ». Mais en 2006, cette prudence s'est un peu étiolée. La rapport de suivi de la PEV sur la Tunisie note :

« en ce qui concerne les droits de l'Homme et les libertés fondamentales, il y a eu peu de progrès des libertés d'association et d'expression. [...] L'exercice de la profession de journaliste continue, dans la pratique, a être soumis à diverses difficultés telles que l'accès des médias à la publicité 96 ».

91. Kelley J., op. cit.

92. Cité par ibid., p. 45.

93. Voir Kingdon J., Agenda, alternatives and public policies, Boston, Little Brown, 1984.

94. Commission des Communautés européennes, Document de travail des services de la Commission. Politique européenne de voisinage, Rapport sur la Tunisie, COM(2004) 373, p. 6.

95. Ibid., p. 8.

96. Commission des Communautés européennes, Document de travail des services de la Commission accompagnant la «Communication from the Commission to the Council and the European Parliament on Strengthening the European Neighbourhood Policy », Rapport 
Comment, face à ce qui vient d'être dit, amener les Etats à respecter les engagements pris dans le cadre des plans d'action? La solution n'est pas simple, loin s'en faut. Interrogés sur l'efficacité de la conditionnalité et des améliorations à y apporter le cas échéant, les officiels de la Commission n'ont d'autre recette que d'insister sur le dialogue et la création des souscomités supplémentaires pour amener les partenaires méditerranéens à passer à la phase politique des plans d'action ${ }^{97}$. Aussi pourrait-on affirmer que jamais la puissance civile de l'UE n'a été dans une telle contradiction. Au total, le complexe méditerranéen de sécurité est dominé par le secteur économique, une faible conviction des Etats-membres et une conditionnalité piégée, à son tour, par la peur d'externalités de sécurité supérieures à celle qui le traversent actuellement.

\section{L'Europe de l'Est}

$\mathrm{Du}$ point de vue géographique, ce complexe s'articule autour de la Moldavie, l'Ukraine et la Biélorussie. L'importance de ce complexe doit beaucoup à l'action de la Pologne, mais aussi à l'urgence pour l'Union de stabiliser et de contribuer au développement politique et économique de la région. Enfin, la région de l'Europe de l'Est tire sa centralité du fait que s'y noue l'enjeu stratégique de l'énergie ou, plus grossièrement, du rapport à la Russie 98 . La Direction générale Energie et Transport de la Commission atteste de fait l'urgence énergétique :

«Si aucune action n'est prise dans les vingt ou trente prochaines années $[\ldots]$ notre dépendance énergétique s'élèvera à $70 \%$ en moyenne, atteignant les $90 \%$ dans le cas des produits pétroliers. Cette situation nous rend vulnérables, surtout si l'on considère la dépendance de notre économie par rapport à certains types d'énergies, tels que le pétrole et le gaz, et par rapport à certains pays tels que la Russie, pour ce qui est du gaz naturel $[\ldots]{ }^{99}{ }^{»}$.

L’Ukraine est, elle aussi, un partenaire essentiel dans le domaine énergétique, en tant que pays de "transit stratégique [...] notamment en ce qui concerne le transit des approvisionnements russes en gaz et en pétrole $100 »$.

suivi Tunisie, COM (2006) 726, p. 3. http//:ec.europa/world/enp/documents_fr.htm

97. Kelley J., op. cit.

98. Voir Delcour L., «La politique européenne de voisinage et les relations russo-européennes : partenariat stratégique ou lutte d'influence?", pp. 7-15. Texte disponible sur: http://www.etudes-européennes.fr

99. Commission of the European Communities, Energy. Let's Overcome our Dependence, 2002, pp. 2-3.

100. Commission des Communautés européennes, Document de travail des services de la Commission accompagnant la «Communication from the Commission to the Council and the European Parliament on Strengthening the European Neighbourhood Policy », Rapport de suivi Ukraine, COM (2006) 726, p. 15. 
En Europe de l'Est, contrairement à la région méditerranéenne, la conduite de la politique européenne a été plus équilibrée. Peut-être est-ce dû au fait que les membres de ce versant de la PEV nourrissent - à tort ou à raison - une réelle ambition d'intégration. Toujours est-il que l'UE n'a pas hésité, chaque fois que l'occasion s'est présentée, à évoquer avec ses partenaires la question des droits de l'Homme et de la démocratie. Ainsi, le régime moldave a plusieurs fois été tancé pour son manque de réforme et, depuis 1987, l'UE lie toute évolution de ses rapports avec la Biélorussie à de profondes transformations institutionnelles, alignées sur les principes de la règle de droit. Il n'est pas téméraire de suggérer, pour en terminer avec ce complexe, qu'il est déficitaire dans les trois piliers de la PEV : du point de vue de la sécurité (conflit en Moldavie, autocratie en Biélorussie); du point de vue de la prospérité (situation économique et sociale très précaires commune aux trois Etats); et du point de vue de la stabilité (crises politiques récurrentes en Ukraine depuis les élections du 26 décembre 2004). La différence essentielle entre le complexe régional méditerranéen et le complexe régional d'Europe de l'Est tient surtout en l'engagement plus résolu de l'UE et en un fort équilibre d'intervention dans les trois secteurs de la politique européenne de voisinage.

\section{Le Caucase}

Difficile à cerner, hétéroclite dans sa composition, cette région est en effet constituée d'Etats que l'on peut répartir par rapport à l'UE comme suit : membres (Grèce, Bulgarie, Roumanie); candidat (Turquie); non membres (Russie, Georgie). L'implication de l'UE y est plus timide, en dépit d'un embargo sur les armes imposé à l'Azerbaïdjan et à l'Arménie. Mais les choses semblent évoluer depuis la mise sur l'agenda européen de la sécurité énergétique. Dans ce contexte, l'Azerbaïdjan occupe une position cruciale. Le Document de Stratégie par Pays affirme :

«l'Azerbaïdjan a, en tant que producteur de pétrole et de gaz et pays de transit, un rôle essentiel à jouer dans [le domaine de la sécurité et de l'approvisionnement énergétique], y compris l'ouverture de voies alternatives de transport pour les ressources énergétiques de la mer Caspienne et de l'Asie centrale vers l'Europe. L'UE a tant des intérêts commerciaux directs que des intérêts politiques à encourager la coopération régionale $[\ldots]^{101}$ ».

Du point de vue politique, l'UE soutient la société civile, qui a eu un rôle dans la révolution rose de Georgie en 2003. L'Union « vise aussi à stabiliser toute la région $d u$ Caucase $d u$ Sud en soutenant le règlement $d u$ conflit dans le Haut-Karabakh entre l'Arménie et l'Azerbaïdjan 102 ». Le tableau n'est cepen-

101 . Instrument européen de voisinage et de partenariat, Azerbaïdjan - Document de stratégie parpays, 2007-2013, p. 5.

102 . Ibid. 
dant pas si positif. Plus généralement, il suffirait de mentionner le contenu d'un document de travail de TACIS qui souligne, à propos de la région :

«Il y a eu très peu de progrès réels en faveur de la démocratisation et du respect des droits des l'homme ; en sens contraire, il y a eu une tendance générale à l'autoritarisme, à la corruption administrative et judiciaire. La société civile demeure faible et la marginalisation des femmes a augmenté dans la région 103 ».

En d'autres termes, à l'instar du complexe de sécurité précédent, celui du Caucase est dominé par la problématique sécuritaire, et plus nettement par la question de l'approvisionnement énergétique. Aussi ne s'étonnera-t-on pas des critiques particulièrement mesurées de l'UE à l'égard de l'Azerbaïdjan, son principal partenaire commercial dans la région :

« Ce système institutionnel semble bien correspondre à un pays qui, depuis son indépendance, a toujours eu des régimes légèrement autoritaires basés sur un mélange complexe d'allégeance politique, de solidarité de clan et d'intérêts économiques ${ }^{104}$ ».

Au total, la meilleure façon de résumer notre propos consiste-t-elle probablement à présenter le complexe de sécurité régional du Caucase comme une expression distincte du dilemme du beurre et du canon.

L'intérêt que l'UE porte aux problèmes qu'elle abandonnait naguère aux voisins, son souci de préciser les conditions dans lesquelles une coopération a des meilleures chances de succès, marquent un tournant stratégique. C'est dire, en d'autres termes, que la $\mathrm{PEV}$, pour peu que l'on y regarde de près, témoigne non sans difficulté de l'émergence de l'Union comme acteur des relations internationales, notamment à travers le rôle que continue d'y jouer la Commission. Mais cette implication de la Commission a ses revers : très élaborée en ses aspects procéduraux et techniques, la PEV est plus incertaine en son volet politique et opérationnel. Le principe de cette indécision a pour assise, comme nous l'avons constaté, trois catégories de problèmes interdépendants que l'on appellera par commodité de langage l'ambiguité, la finalité et l'asymétrie ${ }^{105}$.

\section{L'ambiguïté}

L'objectif de la PEV conserve une indétermination décisive; elle est perçue et vécue à la fois comme un accélérateur d'adhésion et comme un cadre pour le par-

103. On peut retrouver le document sur : http://www.europa.eu.int/external_relations/consultations/cwsp_tacis.htm

104 . Instrument européen de voisinage et de partenariat. Azerbaïdjan - Document de stratégie par pays, op. cit., p. 10. Nous soulignons.

105 . Tassinari F., op. cit. 
tenariat renforcé, sans ouverture sur l'intégration. Cette ambiguité est logée, c'est là le point le plus important, au cœur même des idées et des mécanismes de la PEV puisque le groupe d'Etats qui en constitue la cible est extrêmement hétérogène. Entre les Etats comme l'Ukraine qui pourraient légitimement en vertu de l'article 49 TUE prétendre à l'intégration, et le Maroc dont la candidature fut rejetée en 1987, il y a finalement peu de choses en commun, excepté ce que l'Union décrit comme une absence de prospérité. Certes dans le cas du Maroc les choses sont relativement claires, mais quid d'autres Etats (Moldavie, Biélorussie, Israël et l'Autorité Palestinienne) ? En somme, la simple possibilité d'adhésion, tant que l'UE ne débrouille pas la compétition des ambitions des uns et des autres, prive la PEV de sa spécificité - création d'un cercle d'amis, sans adhésion à la clé.

\section{La finalité}

Par sa composition particulièrement hétérogène, la PEV voudrait combiner deux modes de gouvernance, le holisme et la différenciation. Le premier considère les partenaires comme un ensemble homogène, sous leurs aspects économiques, sociaux et politiques. A contrario, la différenciation insiste sur le caractère unique de chaque partenaire. Or il nous semble que l'équilibre entre holisme et différenciation n'est viable qu'au prix d'une clarification des objectifs de la PEV; ce qui, en dernière instance, nécessite que l'ambiguité qui entoure sa finalité soit levée pour chacun des membres.

\section{L'asymétrie}

Tout semble indiquer que, loin d'être une politique construite avec les voisins, la PEV est une stratégie pour les voisins, ou à destination des voisins ${ }^{106}$. Le mécanisme qui gouverne les rapports entre l'UE et ses voisins est, bien sûr, celui de la conditionnalité ou, par euphémisme, de la socialisation (forcée). Tout dépend en effet des efforts fournis par le partenaire et de la conformité de son comportement à ce que l'Union a prescrit dans le plan d'action. En regard de quoi, la conditionnalité place l'UE dans une position contractuelle de contrôle quasi absolu. En un mot, alors que la Commission insiste sur l'appropriation commune, la réciprocité et le partenariat renforcé, la conditionnalité définit les critères, énonce le rythme et fixe les limites de la coopération ${ }^{107}$. C'est dire, sous une autre forme, que l'orientation de la PEV conduit - condamne même - à adopter une position paradoxale entre d'une part, l'inclusion normative et économique, et d'autre part l'exclusion politique et institutionnelle. Cette tension structurante énonce très précisément le processus d'émergence et de fonctionnement d'un complexe de sécurité à géométrie variable, celui justement dont nous avons tenté d'esquisser les contours et les effets. 